\title{
A local variable model for entanglement swapping exploiting the detection loophole
}

\author{
Nicolas Gisin and Bernard Gisin \\ Group of Applied Physics, University of Geneva \\ 20, rue de l'Ecole-de-Médecine, CH-1211 Geneva 4, Switzerland
}

\begin{abstract}
In an entanglement swapping process two initially uncorrelated qubits become entangled, without any direct interaction. We present a model using local variables aiming at reproducing this remarkable process, under the realistic assumption of finite detection efficiencies. The model assumes that the local variables describing the two qubits are initially completely uncorrelated. Nevertheless, we show that once conditioned on the Bell measurement result, the local variables bear enough correlation to simulate quantum measurement results with correlation very close to the quantum prediction. When only a partial Bell measurement is simulated, as carried out is all experiments so far, then the model recovers analytically the quantum prediction.
\end{abstract}

\section{INTRODUCTION}

Quantum theory is nonlocal and the richness of entanglement impressive. For the simplest possible case of a two qubits system, the states can be divided into:

1. the separable ones: states that can be prepared locally and whose correlation can be described locally,

2. the poorly entangled ones: states that can't be prepared locally but which admit a local hidden variable model, i.e. the correlation between any projective measurement can be described locally,

3. the fully entangled ones: states that can't be prepared locally and whose correlation - even between projective measurements - can't be described locally.

To this list one should add the class of states useful for teleportation [1] and the ones that can be distilled (using 1- or 2-way classical communication). One might also need to consider generalized measurements (POVMs) [2]. In higher dimensions it is known that these structures are even more involved. Similarly, when considering systems with more than two subsystems further classes are necessary to classify the states (i.e GHZ- and W-states for 3 qubits [3]).

In this letter we consider the class of states that can be experimentally demonstrated to be nonlocal. This class depends on the efficiency of available detectors. The problem may thus seem independent from the entanglement classification program, but this is not really so for at least two reasons. First, this program being part of physics, it must lead to experimental tests. For example, the possibility to experimentally demonstrate entanglement is probably the best - if not the only - way to convince a doubtful customer that a pair of devices constitute the source and receiver of a truly quantum cryptographic system (i.e. that they are really quantum and not merely fakes!) [4,5]. Quantum error correction and linear optics quantum computation provide further examples: for each such schemes to operate, each component must surpass a certain threshold in efficiency. A second motivation for the question we address is its connection to the timely problem of communication complexity [6]. Indeed, every local model mimicking quantum correlation for a detection efficiency $\eta$ can be associated to a model for perfect detectors but for a more noisy state: a mixture of the original state and of a fraction of the maximally mixed state. The association works as follows: in each instance where the original model assumes that the detector fails to register a count, the corresponding model assumes that a random (i.e. uncorrelated) output is produced 1 .

For 2-qubit systems, the problem of demonstrating quantum non locally via violation of the Bell inequality has a long history. Pearle, Shimony and Clauser seem to have been the first to notice the detection loophole, latter studied in details by Santos and others [7]. This loophole resisted to experimenters until last year when the Boulder group published results obtains with detectors sufficiently efficient to close it (but with only $3 \mathrm{mi}-$ crons separation, so that quantum nonlocality is still not demonstrated as beautifully as one would like for such a fundamental concept!) 9].

This letter is organized as follows. First, in the next section, we briefly review the detection loophole and how a simple local hidden variable model can exploit it. Next, we present the basic intuition behind this letter: in entanglement swapping [10] it is very natural to assume that the two input photon pairs are independent (recall that, in principle they are produced by two independent

\footnotetext{
${ }^{1}$ This applies also to the model we presented in a previous publication [8]. In this case, either one detector has an efficiency limited to $50 \%$, or the maximally entangled 2-qubit state is mixed with $50 \%$ of the maximally entangled state, i.e. is the Werner state. At the time of writing our previous model, we where not aware of the close connection between our model and Werner's local hidden variable model.
} 
sources). Consequently, any assumed local variables associated to the qubits, after the entangled swapping process has completed, must be uncorrelated, or at least only poorly correlated. This reduced correlation should make it more difficult to simulate the quantum correlation, hence should make it easier to close the detection loophole. This intuition is expanded in section III. Finally, two explicit models are presented and analysed. The first model assumes a partial Bell measurement (section [V], the second one a complete Bell measurement but always with a finite detection efficiency (section $\mathrm{V}$ ).

\section{REMINDER OF THE DETECTION LOOPHOLE AND A RELATED SIMPLE MODEL OF QUANTUM CORRELATION}

The idea behind the detection loophole is very simple and natural. It merely states that the probability that a particle is detected depends, among others, on the particle's state. This is true as well in classical as in quantum physics. There is thus no reason to reject the assumption that the same would be true in some hypothetical theory which would incorporate additional variables $\lambda$ 's, whether local or not. But then, if the additional variables $\lambda$ are not under the physicist's control (i.e. are hidden), the detection probability may vary from one run of the experiment to the next. This simple idea allows one to construct a straightforward and explicit model reproducing the quantum correlation exactly, using only local variables [8].

It is useful to remind this simple model here, since the entanglement swapping models of the next sections are strongly inspired by it (but for the proofs we refer the reader to 8 ). The model uses the Poincaré sphere $S^{2}$ representation of the qubit state space. Here we remind the simplest model, reproducing the correlation of the quantum singlet state. This model treats the two qubits asymmetrically, but it is straightforward to symmetries the model 87. The jth qubit's state is described by a normalized vector $\vec{\lambda}_{j}, \mathrm{j}=1,2$. When the first qubit is measured along direction $\vec{a}$ (i.e. the operator representing the measured quantity is $\vec{a} \vec{\sigma}$, with $\vec{\sigma}$ the Pauli matrices), then the probability that the particle carrying the qubit is not detected is $1-\left|\vec{a} \vec{\lambda}_{1}\right|$, and if the particle is detected, then the outcome is $\operatorname{sign}\left(\vec{a} \vec{\lambda}_{1}\right)$. When the second qubit is measured along direction $\vec{b}$, it always produces an outcome equal to $\operatorname{sign}\left(\vec{b} \vec{\lambda}_{2}\right)$. Assuming that the qubit pair source produces random $\vec{\lambda}_{1}$, but with $\vec{\lambda}_{2}=-\vec{\lambda}_{1}$, this model predicts a mean detection efficiency (averaged over all $\vec{\lambda}$ 's and over all detectors) of 0.75 and a correlation (conditioned on both qubits producing an outcome) equal to the quantum prediction: $E(\vec{a}, \vec{b})=-\vec{a} \vec{b}$ ! This rather simple and natural (in the spirit of local hidden variables) model proves that no experiments on two maximally entangled qubits with detector efficiencies smaller than $75 \%$ can definitively demonstrate quantum nonlocality, whatever the number of settings and measurements used on each side (the $\mathrm{CH}$ inequality 11 proves that when restricted to two settings on each side, the minimal detection efficiency using qubits in the singlet state is even slightly larger 2 : $82.8 \%$ ).

\section{THE INTUITION}

The task of an experimental demonstration of quantum nonlocality without loopholes requires to surpass some technological thresholds. There is little doubt that this will be achieved in the future, but there is clearly also the desire to simplify the experiment by clever reasoning 12]. One possibility is to go to higher dimensional systems. Indeed, Serge Massar showed that the required detection efficiency decreases exponentially with the dimension when two system are maximally entangled [13]. Here we explore another idea, related to entanglement swapping [10]. This very remarkable process allows two qubits that never interacted to become entangled via an interaction which affects only two other qubits, each of the latter being initially entangled with one of the former qubits, see figure 1 . When trying to simulate this remarkable process with local physics, it is very natural to assume that the local variables $\lambda_{1}=-\lambda_{2}$ characterizing the first entangled qubit pair is independent of the local variable $\lambda_{3}=-\lambda_{4}$ characterizing the second entangled qubit pair. Consequently, after the entanglement swapping, i.e. after one qubit of each pair (e.g. qubits number 2 and 3) interacted in a measurement process, the two remaining qubits are characterized by $\lambda_{1}$ and $\lambda_{4}$, respectively. Since $\lambda_{1}$ and $\lambda_{4}$ are independent, the two remaining qubits are uncorrelated, in agreement with the quantum prediction. However, quantum mechanics predicts that when the state of qubits number 1 and 4 is conditioned on the result of the measurement carried out on their twins (i.e. on qubits 2 and 3), then the qubits 1 and 4 are fully entangled. Since the measurement has 4 possibly outcomes, the set of variables $\left(\lambda_{2}, \lambda_{3}\right)$ must be divided into 5 subsets: 4 subsets corresponding to the 4 possible measurement outcomes and the fifth one to the case of no-detection. In each subset $\lambda_{2}$ and $\lambda_{3}$ may have some correlation, but clearly not full correlation, in particular not of the form $\lambda_{2}=-\lambda_{1}$ as in the simple model recalled in the previous section. Hence, it is intuitively clear that an experiment can rule out such local models with lower detection efficiencies than required in standard Bell tests. But we shall see that the improvement

\footnotetext{
${ }^{2}$ Whether this difference means that there is a better local model or a better Bell inequality - e.g. involving more than two settings by site or using generalized measurements - is an interesting open question.
} 
is surprisingly small.

\section{A FIRST MODEL: CASE OF PARTIAL BELL-MEASUREMENT}

In this first model of entanglement swapping we assume that only an incomplete Bell measurement is performed on the two central qubits number 2 and 3 , see figure 1. This partial Bell measurement only singles out the singled state, as is the case in all experiments today [14. In this model each of the 4 qubit states are described by normalized vectors $\vec{\lambda}_{j}, \mathrm{j}=1,2,3,4$. The two qubit pair sources produce random $\vec{\lambda}$ 's, but with $\vec{\lambda}_{2}=-\vec{\lambda}_{1}$ and $\vec{\lambda}_{4}=-\vec{\lambda}_{3}$, in agreement with the simple model recalled in section II. The first entanglement swapping model assumes that the partial Bell measurement produces the singlet-outcome iff

$$
\vec{\lambda}_{2} \cdot \vec{\lambda}_{3} \leq 1-\frac{\eta^{2}}{2},
$$

where $\eta$ is a parameter of the model characterizing the averaged detection efficiency $(0 \leq \eta \leq 1)$. Accordingly, the probability of the singlet-outcome reads:

$$
\begin{aligned}
P(\text { singlet }) & =\int_{S^{2}} \int_{S^{2}} \frac{d \vec{\lambda}_{2} d \vec{\lambda}_{3}}{(4 \pi)^{2}} \theta\left(1-\frac{\eta^{2}}{2}-\vec{\lambda}_{2} \vec{\lambda}_{3}\right) \\
& =\int_{-1}^{1} \frac{d z}{2} \theta\left(1-\frac{\eta^{2}}{2}-z\right) \\
& =\frac{\eta^{2}}{4}
\end{aligned}
$$

where $\theta$ is Heavyside's function. Hence, as announced, the model predicts that the partial Bell measurement produces the outcome singlet with the same probability as quantum mechanics when both qubits are detected with an efficiency $\eta$ (the factor $\frac{1}{4}$ takes into account that in a partial Bell measurement only 1 of the 4 Bell states is detected, even in principle). Now, the model is exactly as in section II: when the first qubit is measured along direction $\vec{a}$ it produces the result $\operatorname{sign}\left(\vec{a} \vec{\lambda}_{1}\right)$ with probability $\left|\vec{a} \vec{\lambda}_{1}\right|$ and no outcome at all with the complementary probability. The last qubit, when measured along $\vec{b}$ always produces the result $\operatorname{sign}\left(\vec{b} \vec{\lambda}_{4}\right)$. Let us stress that the asymmetry between qubit 1 and 4 in this model can be remove by adding auxiliary random variables, similarly to 8 .

Let us now compute the predictions of this model. First, the probability that the partial Bell measurement produces the outcome singlet and that the first qubit produces an outcome, i.e the probability that all the 4 qubits fire a detector, reads (using $\vec{\lambda}_{2}=-\vec{\lambda}_{1}$ ):

$$
\begin{aligned}
p & \equiv \operatorname{Prob}(\text { Bell }=\text { singlet } \& a \neq 0) \\
& =\int_{S^{2}} \int_{S^{2}} \frac{d \vec{\lambda}_{1} d \vec{\lambda}_{3}}{(4 \pi)^{2}}\left|\vec{a} \vec{\lambda}_{1}\right| \theta\left(\vec{\lambda}_{1} \vec{\lambda}_{3}-\left(1-\frac{\eta^{2}}{2}\right)\right)
\end{aligned}
$$

$$
\begin{aligned}
& =\int_{S^{2}} \frac{d \vec{\lambda}_{1}}{4 \pi}\left|\vec{a} \vec{\lambda}_{1}\right| \frac{1-\left(1-\eta^{2} / 2\right)}{2} \\
& =\frac{\eta^{2}}{8}
\end{aligned}
$$

Next, the distribution probability of $\vec{\lambda}_{1}$ and $\vec{\lambda}_{4}$ conditioned of all qubits being detected reads:

$$
\begin{array}{r}
\rho\left(\vec{\lambda}_{1}, \vec{\lambda}_{4} \mid \text { Bell }=\text { singlet \& } a \neq 0\right)= \\
=\frac{\frac{1}{(4 \pi)^{2}}\left|\vec{a} \vec{\lambda}_{1}\right| \theta\left(1-\frac{\eta^{2}}{2}-\vec{\lambda}_{1} \vec{\lambda}_{4}\right)}{p}
\end{array}
$$

Finally, the correlation function when qubits 1 and 4 are measured along directions $\vec{a}$ and $\vec{b}$, conditioned on all qubits being detected, reads:

$$
\begin{aligned}
E(\vec{a}, \vec{b}) & =\int_{S^{2}} \int_{S^{2}} d \vec{\lambda}_{1} d \vec{\lambda}_{4} \rho\left(\vec{\lambda}_{1}, \vec{\lambda}_{4} \mid \text { Bell }=\text { singlet } \& a \neq 0\right) \\
& =\frac{1}{2 \pi^{2} \eta^{2}} \int_{S^{2}} \int_{S^{2}} d \vec{\lambda}_{1} d \vec{\lambda}_{4} \theta\left(1-\frac{\eta^{2}}{2}-\vec{\lambda}_{1} \vec{\lambda}_{4}\right) \cdot \\
& =\frac{1}{2 \pi^{2} \eta^{2}} \int_{S^{2}} d \vec{a} \vec{\lambda}_{1} \operatorname{sign}\left(\vec{b} \vec{\lambda}_{4}\right) \operatorname{sign}\left(\vec{b} \vec{\lambda}_{4}\right) \cdot \\
& \cdot \int_{S^{2}} d \vec{\lambda}_{1} \theta\left(1-\frac{\eta^{2}}{2}-\vec{\lambda}_{1} \vec{\lambda}_{4}\right) \vec{a} \vec{\lambda}_{1} \\
& =\frac{1-\left(1-\eta^{2} / 2\right)^{2}}{2 \pi \eta^{2}} \int_{S^{2}} d \vec{\lambda}_{4} \operatorname{sign}\left(\vec{b} \vec{\lambda}_{4}\right) \vec{a} \vec{\lambda}_{4} \\
= & -\left(1-\frac{\eta^{2}}{4}\right) \vec{a} \vec{b}
\end{aligned}
$$

This model of entanglement swapping with a partial Bell measurement predicts thus correlations of exactly the same form as the quantum prediction, but with a reduced visibility

$$
V=1-\frac{\eta^{2}}{4}
$$

Consequently - and surprisingly! - using entanglement swapping with a partial Bell measurement barely helps demonstrating quantum nonlocality. Indeed, one would need to obtain a measured correlation between the initially independent two qubits with a visibility larger than $75 \%$, i.e. larger than the usual Bell-threshold of $70.7 \%$ (this usual threshold does not take into account the detector loophole which we consider here). Actually, with realistic Bell measurements of finite efficiency, the required visibility is even higher, as indicated in (15)! For example, for realistic photon counters with $\eta \approx 0.4$, one has $V \approx 94 \%$.

Let us stress the surprising feature of this model. Initially the two local variables $\vec{\lambda}_{1}$ and $\vec{\lambda}_{4}$ are completely independent. Even when conditioned upon the partial Bell measurement outcome singlet, these two local variables 
are only poorly correlated. Nevertheless the correlation predicted from these poorly correlated local variables has the quantum mechanical shape, with merely a reduced visibility (note that this model, like the one in section II, assumes a mean detection efficiency smaller or equal to $75 \%$ ). Moreover, for realistic detection efficiencies the visibility is only slightly below 1 . Consequently, no experiments on entanglement swapping experiments with partial Bell measurement and realistic detector efficiencies can definitively demonstrate quantum nonlocality, whatever the number of settings and measurements used on each side!

\section{SECOND MODEL: CASE OF COMPLETE BELL-MEASUREMENTS}

In the light of the rather disappointing result of the previous section, it is tempting to investigate the case of entanglement swapping with a complete Bell measurement. Indeed, it is intuitively clear that in such a case the Bell measurement can't correlate the local variables $\vec{\lambda}_{1}$ and $\vec{\lambda}_{4}$ as much as in the first model. The idea of this second model is closely inspired by the previous one and by the well known fact that all the 4 Bell states can be obtained from the singlet one, $\psi^{(-)}$, with only one local rotations:

$$
\begin{aligned}
\phi^{(-)} & =\mathbb{1} \otimes \sigma_{x} \psi^{(-)} \\
\phi^{(+)} & =\mathbb{1} \otimes \sigma_{y} \psi^{(-)} \\
\psi^{(+)} & =\mathbb{1} \otimes \sigma_{z} \psi^{(-)}
\end{aligned}
$$

Hence, the idea is that the Bell measurement results should produce the following correlation:

$$
\begin{aligned}
& \psi^{(-)} \Rightarrow \vec{\lambda}_{1} \approx-\vec{\lambda}_{4} \\
& \phi^{(-)} \Rightarrow \vec{\lambda}_{1} \approx-R_{x} \vec{\lambda}_{4} \\
& \phi^{(+)} \Rightarrow \vec{\lambda}_{1} \approx-R_{y} \vec{\lambda}_{4} \\
& \psi^{(+)} \Rightarrow \vec{\lambda}_{1} \approx-R_{z} \vec{\lambda}_{4}
\end{aligned}
$$

where $R_{k}$ represents a $\pi$-rotation around the k-axis. Following this idea, the second model assumes that the Bell measurement result is the one corresponding to the most negative scalar product among the following four: $\vec{\lambda}_{1} \cdot \vec{\lambda}_{4}, \vec{\lambda}_{1} \cdot R_{x} \vec{\lambda}_{4}, \vec{\lambda}_{1} \cdot R_{y} \vec{\lambda}_{4}$ and $\vec{\lambda}_{1} \cdot R_{z} \vec{\lambda}_{4}$, provided it is lower than -Limit, where Limit is a parameter which determines the Bell measurement efficiency, see Fig. 2.

For this second model, we were unable to find the analytic form of the predicted correlation. However, simple numerical simulations can be used. Fig. 3 presents the mean fidelity as estimated by the correlation when both analysers measuring qubits 1 and 4 are parallel. Finally, Fig. 4 and 5 illustrate the simulated correlation when Alice's analyser is fixed and Bob's one rotated. On each graph there are 4 correlation corresponding to the $2 \mathrm{x} 2$ possible results on Alice and Bob sites together with 4 sinusoidal fits. These numerical results, and many other not shown here, demonstrate that the shape of the correlation is no longer strictly proportional to the quantum one: $E(\vec{a}, \vec{b}) \neq c s t \cdot \vec{a} \vec{b}$. However, the difference is so small that it is still essential impossible to find a measurable difference!

\section{CONCLUSION}

Quantum nonlocality is fundamental for the wordview offered by physics and central for several applications of quantum information theory. Consequently, it deserves to be carefully tested. The detection loophole is annoying because it resists almost all experimental efforts (see however [9]). It is also fascinating because of the connections mentioned in the introduction with quantum cryptography and with quantum communication complexity: it offer a nice example of the close connection between basic and applied physics. In this letter we have shown that even for a process like entanglement swapping, where entanglement is established between particle that never interacted directly, the detection loophole remains exceedingly difficult to close.

\section{ACKNOWLEDGMENTS}

Support by the Swiss FNRS and NCCR Quantum Photonics are acknowledged.

[1] J. Barrett, Phys. Rev. A 64, 042305, 2001.

[2] J. Barrett, A local hidden variable model for positive operator valued measurements on a class of entangled mixed states, Quant-ph/0107045.

[3] W. Dür, G. Vidal and I. Cirac, Phys. Rev. A 62, 062314, 2000.

[4] D. Mayers and A. Yao, Quantum Cryptography with Imperfect Apparatus, Proceedings of the 39th IEEE Conference on Foundations of Computer Science, 1998.

[5] N. Gisin, G. Ribordy, W. Tittel and H. Zbinden, Quantum Cryptography, quant-ph/0101098, in press, Rev. Modern Phys., 2002.

[6] G. Brassard, Quantum communication complexity, Quant-ph/0101005.

[7] P. Pearle, Phys. Rev. D, 2, 1418, 1970; J.F. Clauser, M.A. Horne, A. Shimony, and R.A. Holt, Phys. Rev. Lett., 23, 880, (1969); E. Santos, Phys.Rev. A, 46, 3646, 1992.

[8] N. Gisin and B. Gisin, Phys. Lett. A 260, 323-327, 1999.

[9] M.A. Rowe et al., Nature 409, 791, 2001.

[10] M. Żukowski, A. Zeilinger, M.A. Horne and A. Ekert, Phys. Rev. Lett. 71, 4287, 1993. 
[11] J.F. Clauser and M.A. Horne, Phys. Rev. D 10, 526, 1974.

[12] N. Gisin and H. Zbinden, Phys. Lett. A 264, 103, 1999.

[13] S. Massar, ???

[14] J.-W. Pan, D. Bouwmeester, H. Weinfurter and A. Zeilinger, Phys. Rev. Lett. 80, 3891, 1998; Th. Jennewein, G. Weihs, J.-W. Pan and A. Zeilinger, Phys. Rev. Lett. 88, 017903, 2002.

\section{FIGURE CAPTIONS}

1. General scheme of the entanglement swapping setup. Each 2-photon source produces qubit pairs in the singlet state. In the local variable model under consideration the state of each qubit is entirely characterized by a normalized 3-dim vector, i.e. a point on the Poincaré sphere, each randomly distributed on the entire sphere. The sources produce correlated qubits with local parameters $\vec{\lambda}_{2}=-\vec{\lambda}_{1}$ and $\vec{\lambda}_{4}=-\vec{\lambda}_{3}$. The 2 sources are independent, consequently $\vec{\lambda}_{1}$ and $\vec{\lambda}_{4}$ are statistically independent. The Bell measurement produces one of 5 possible outcomes: one of the 4 usual Bell states or the nodetection result. In the local variable model the probability for these 5 outcomes depend only on $\vec{\lambda}_{2}$ and $\vec{\lambda}_{3}$. In the simplified model (section [V]) only a partial Bell measurement is considered, i.e. only 2 outcomes are considered: either singlet, or no-detection.

2. Numerical simulation of the second model, assuming a complete Bell measurement (on $10^{8}$ samples). The probability that the Bell measurement gives a result is computed in function of the parameter Limit (see text).

3. The fidelity of the entanglement swapping simulation is computed (on $10^{7}$ samples) in function of the probability that the complete Bell measurement gives a result.

4. The 4 correlation function between the results on Alice and on Bob sites (two possible outcomes per site) are computed (on $10^{6}$ samples) in function of a rotation of Bob's analyser. The efficiency of the Bell measurement is assumed at $\approx 50 \%$. Four sinusoidal fits are also displayed, illustrating that the model, although different from the quantum prediction, is in practice undistinguishable.

5. Same as 4, but for different analyser setting and a Bell measurement efficiency $\approx 90 \%$. 